Abelian sandpile model on the Bethe lattice

This article has been downloaded from IOPscience. Please scroll down to see the full text article.

1990 J. Phys. A: Math. Gen. 234333

(http://iopscience.iop.org/0305-4470/23/19/018)

View the table of contents for this issue, or go to the journal homepage for more

Download details:

IP Address: 129.175.97.14

The article was downloaded on 05/01/2011 at 10:27

Please note that terms and conditions apply. 


\title{
Abelian sandpile model on the Bethe lattice
}

\author{
Deepak Dhar and S N Majumdar \\ Tata Institute of Fundamental Research, Homi Bhabha Road, Bombay 400005, India
}

Received 4 April 1990

\begin{abstract}
We study Bak, Tang and Wiesenfeld's Abelian sandpile model of selforganised criticality on the Bethe lattice. Exact expressions for various distribution functions including the height distribution at a site and the joint distribution of heights at two sites separated by an arbitrary distance are obtained. We also determine the probability distribution of the number of distinct sites that topple at least once, the number of topplings at the origin and the total number of topplings in an avalanche. The probability that an avalanche consists of more than $n$ topplings varies as $n^{-1 / 2}$ for large $n$. The probability that its duration exceeds $T$ decreases as $1 / T$ for large $T$. These exponents are the same as for the critical percolation clusters in mean-field theory.
\end{abstract}

\section{Introduction}

In recent years, the concept of self-organised criticality (SOC) proposed by Bak, Tang and Wiesenfeld (BTW) [1, 2] has generated much interest. There is no generally agreed definition of SOC. We shall adopt the view (see Takayasu et al [3]) that the existence of scale-independent fluctuations without fine tuning of coupling constants is the defining characteristic of SOC. Power-law correlations are encountered very frequently in nature. In some cases, SOC is only a new name for, and perhaps a new way of looking at, a familiar and fairly well understood phenomenon. An example is the low-temperature phase of the $n$-vector model for $n \geq 2$ in $d>2$. In this case, it is well known that, in the absence of an external field, transverse fluctuations of magnetisation have power-law correlations in the entire low-temperature phase in equilibrium [4]. It seems reasonable that for most local dynamical evolution rules (stochastic or deterministic), these would also imply a power-law decay of time-dependent correlations. The criticality in this case is related to the existence of slowly decaying long wavelength (Goldstone) modes in the problem. The importance of the role of Goldsone modes in SOC has been noted earlier $[5,6]$.

There are power-law tails in the velocity autocorrelation function in fluids (and in solids) also. Thus, from our point of view, all fluids show SOC behaviour, as shown by the existence of hydrodynamic modes (phonons for solids) in the system. In turbulence also, criticality is due to the existence of hydrodynamical modes, but in this case, the critical exponents take non-trivial values due to the strong coupling between different modes [7].

A somewhat similar example of SOC is the steady state of an ecological system having $N$ species and with a population dynamics of the Lotka-Volterra type [8]. Let $C_{i}(r, t)$ be the density of the population of species $i$ at point $r$ at time $t$. We assume 
that the rates of change of the $C_{i}$ are quadratic in the local densities, with additional terms to account for diffusion in space and for stochasticity in birth rates. In the absence of noise, such equations are known to possess spatially uniform solutions oscillatory in time in some parameter regimes. Even with diffusion and stochasticity, clearly long-wavelength perturbations of the phases of oscillations will decay very slowly in time and will lead to slower than exponential decay of correlations in space and time. The forest-fire model proposed by Bak et al [9] is qualitatively similar. In this model, the burning trees and the unburnt trees are the two interacting ecological species (predator and prey).

Another class of problems where SOC in this general sense is encountered is in models of irreversible growth. Kadanoff [5] has pointed out that diffusion-limited aggregates may be called self-organised critical as they have non-trivial power-law exponents independent of the precise value of, say, the sticking probability. Note that diffusion-limited aggregation may also be thought of as a special case of reaction diffusion systems discussed in the previous paragraph. In a similar sense, one could argue that other irreversible aggregation models, e.g. models of gelation [10], the Eden model [11] (for non-trivial surface properties), invasion percolation [12], show SOC.

In fact, a large linear (or branched) polymer molecule in a solvent is also an example of SOC, as the positions of monomers show non-trivial power-law correlation. This example is interesting in that it is a system in thermal equilibrium, and it is at a critical point in the conventional thermodynamic sense. (In the equivalence of this problem to the $n=0$ vector model, the distance from the critical temperature varies as $1 / N$, where $N$ is the number of monomers in the chain.) In a similar way, a system which undergoes a thermodynamical first-order phase transition is self-organised critical in the coexistence region. (One can add, say, $1 \mathrm{gm}$ of ice to a comparable mass of water at roughly room temperature. On stirring these two, the resulting combined system is at the phase coexistence line for a range of values of input parameters.) On the phase coexistence line, it is well known that there are non-exponential tails in correlation functions, as shown by the phenomenon of hysteresis and the existence of power-laws in the late-stage growth of phase separation [13].

Given the wide range of systems which show SOC, it is not surprising that several different models of it have been proposed and studied in the last two years $[9,14,15]$. Of the several models that have been studied, the sandpile automaton model has attracted maximum attention [16-23] because of its intuitive appeal and the simplicity of its mathematical structure [24]. In the case when there is a preferred direction, an exact solution has been obtained and the corresponding exponents are known in all dimensions [25]. The undirected problem also appears to be exactly solvable, as shown by the simple expressions for entropy of the self-organised critical state and the twopoint correlation function obtained earlier [24], but it has so far not been possible to compute quantities such as the distribution function of sizes of avalanches in any dimension other than the trivial $d=1$ case.

In this paper, we study the BTW sandpile model on the Bethe lattice. We assume that the toppling conditions depend on the local height of the sandpile, but not on its gradient. This model has been termed the Abelian model [24] as the operators representing the addition of particles at different sites satisfy a commutative algebra. This Abelian property of the operator algebra is absent in other BTW-type models where the toppling criteria depend on the local gradients of height $[21,26]$. We are able to obtain a fairly detailed characterisation of the SOC state of the Abelian model on the Bethe lattice. We give explicit expressions for the single-site height distribution function, 
the joint distribution function of heights at two sites separated by arbitrary distance $R$ and the distribution of the number of distinct sites toppled in an avalanche. The asymptotic form of the distribution of avalanche durations is also determined. We find these distributions are characterised by the mean-field value of exponents describing cluster distributions in percolation theory at the critical percolation threshold.

The plan of the paper is as follows. In section 2 , we define the model precisely. In section 3 , we introduce the recursion relations to enumerate the stable configurations that are allowed in the SOC state. The enumeration of allowed configurations subject to different constraints allows us to determine various $n$-point correlation functions in the SOC state. In section 4 we compute explicitly the single-site height distribution function. The joint distribution of heights at two sites separated by arbitrary distance is calculated in section 5 . In section 6 , the distribution functions of the mass and duration of avalanches are discussed and the critical exponents determined. Section 7 deals with the distribution functions of the number of topplings at a site and the total number of topplings in an avalanche. In section 8 , we generalise our results to Bethe lattices with other coordination numbers. Section 9 contains some concluding remarks.

\section{Preliminaries}

We consider a Cayley tree of $N$ sites such that all sites of the tree have coordination number 3 , except the boundary sites which have a coordination number 1 .

The BTW model on this lattice is an automation model defined as follows: at each site $i(1 \leq i \leq N)$ we associate an integer $z_{i}\left(1 \leq z_{i} \leq 3\right)$. In sandpile terminology, $z_{i}$ is the height of a sand column at site $i$. The dynamics of the model is defined by two rules.

(i) Adding a particle: if the system is in a stable configuration, we choose at random a site $i$ (all sites equally likely), and increase the height at that site by 1 . Heights at other sites remain unchanged.

(ii) Toppling rule: if at any site the height of the sand column exceeds 3 then that site topples, its height decreases by 3 and the sand particles drop on the nearest neighbours. As a result, the height at each of the nearest neighbours increases by 1. Note that each toppling at sites on the surface decreases the number of particles in the system by 2 , but toppling at non-surface sites conserves the number of (sand) particles.

Adding a particle at a randomly chosen site may cause it to topple, and the toppling may induce toppling at some of the neighbouring sites at the next time step, thus causing an avalanche. Eventually the system reaches a stable configuration (no more topplings). If we keep on adding particles randomly, the system ultimately reaches a statistically stationary state which is critical in the sense that the probability distribution of the size of an avalanche by mass or by duration shows a power-law behaviour for a very wide range of sizes (the upper cut-off on the size is determined by the number of sites of the lattice, and is strictly infinite in the thermodynamic limit).

A well recognised problem with the Cayley tree is that most of its sites are very near to the surface. Hence, calculation of the thermodynamic limit of the 'bulk' properties from the finite $N$ calculation requires special care. Consider a site at a distance $r$ from the surface. If we add a particle at this site, the expected duration 
of the resulting avalanche in the SOC state tends to infinity only when $r \rightarrow \infty$. Thus in order to study the SOC state, we shall only consider the distribution of avalanches caused by adding a particle at a site very far from the surface. This is the procedure we shall use to define the problem on the Bethe lattice [27]. Note that the particles are added at random near the surface also (else the existence of a unique SOC state is not guaranteed). But in calculating the distribution of avalanche sizes, avalanches caused by additions near the surface are not included.

In a stable configuration, at any site $i$ the height of the sand column $z_{i}$ can take three possible values 1,2 or 3 . As the lattice has $N$ sites, the total number of stable configurations is $3^{N}$. But not all of them are allowed in the soc state. For example, if in a configuration, there are two adjacent sites (say $a$ and $b$ ) both having heights 1 (figure $1(a)$ ), then that configuration occurs with zero probability in the SOC state. This is easy to see. Suppose we start with any arbitrary initial configuration with $z_{a} \neq 1$ and $z_{b} \neq 1$ and add particles randomly at different sites. In order to reach a configuration with $z_{a}=1$ and $z_{b}=1$, at least one toppling must have occurred at both the sites. Say $b$ topples last. But any toppling at $b$ increases $z_{a}$ by 1 . Then just before $b$ 's last toppling, $z_{a}$ must be 0 , but this is not allowed in the model. Therefore, we will never reach a configuration with $z_{a}=1$ and $z_{b}=1$. Thus, any configuration with unit heights at adjacent sites is forbidden in the SOC state.

(a)

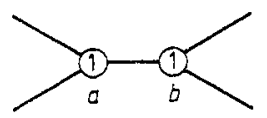

(b)

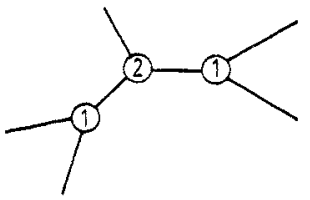

Figure 1. Two examples of configurations that cannot occur in the soC state. Numbers inside the circles are the values of heights at the sites. Symbols just outside the sites are the site labels.

Similarly, one can show that any configuration containing a site with height 2 having two neighbours of height 1 is also forbidden in the SOC state (figure $1(b)$ ).

In general, any set $F$ of $r$ sites $(r \geq 1)$ whose heights $z_{j}(\forall j \in F)$ satisfy

$$
z_{j} \leq \text { coordination number of } j \text { in } F \quad \forall j \in F
$$

is not allowed in the SOC state. We call such a configuration of heights on a finite connected set of sites a forbidden subconfiguration (FSC) [24].

The allowed configurations on a subtree of the Cayley tree can be divided into two classes: weak and strong. These are defined as follows. Let $T$ be a rooted subtree of the lattice with root vertex $a$. Let $T$ be connected to the rest of the lattice via the site $b$ (figure 2(a)). Consider an allowed subconfiguration $C$ on $T$ (i.e. $C$ does not contain any FSC in it). For any height $z_{b}$ at $b$, this defines a subconfiguration $C^{\prime}$ on the tree $T^{\prime}(=T \cup b)$. If $C^{\prime}$ does not contain any FSCs in it even when $z_{b}=1$, then $C$ is called a strongly allowed subconfiguration on $T$. On the other hand, if $C^{\prime}$ becomes forbidden on $T^{\prime}$ if $z_{b}=1$ (though $C$ is allowed on $T$ ), then $C$ is called a weakly allowed subconfiguration on $T$.

As an example, consider an allowed subconfiguration $C$ on a subtree $T$ of three sites shown in figure $2(b)$ with $z_{a}=1$. In this case, if $z_{b}=1$, then in $T^{\prime}(=T \cup b)$, we get an FSC. Hence, $C$ is weakly allowed on $T$, whereas the subconfiguration $z_{a}=2$ with other heights unchanged (figure $2(c)$ ) is strongly allowed on $T$. 


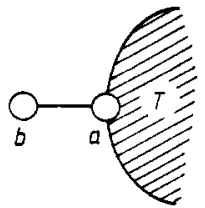

(a)

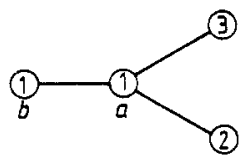

(b)

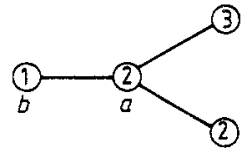

(c)

Figure 2. (a) A subtree $T$ with root vertex $a$. The rest of the tree is denoted by the hatched area. The site $b$ is the nearest neighbour of $a$, but not part of $T$. (b) An example of a weakly allowed subconfiguration on the tree $T$ (consisting of three sites only) with vertex $a$ having unit height (i.e. $z_{a}=1$ ). (c) The same graph as (b) becomes strongly allowed if $z_{a}=2$.

\section{Recursion relations}

Let, $T^{\prime}$ and $T^{\prime \prime}$ be two rooted subtrees of the lattice with root vertices $a^{\prime}$ and $a^{\prime \prime}$ connected by a single bond (figure 3 ). Let, $C^{\prime}$ and $C^{\prime \prime}$ be two allowed subconfigurations on $T^{\prime}$ and $T^{\prime \prime}$ respectively. Then the configuration $C\left(=C^{\prime} \cup C^{\prime \prime}\right)$ on $T\left(=T^{\prime} \cup T^{\prime \prime}\right)$ might be allowed or forbidden depending upon $C^{\prime}$ and $C^{\prime \prime}$. It is easy to verify that $C$ will be forbidden on $T$ iff both $C^{\prime}$ and $C^{\prime \prime}$ are weakly allowed subconfigurations.

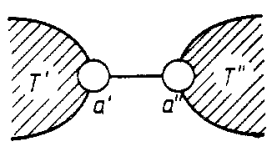

Figure 3. Two adjacent sites $a^{\prime}$ and $a^{\prime \prime}$ are the vertices of two rooted subtrees $T^{\prime}$ and $T^{\prime \prime}$ respectively. A configuration on the tree $T\left(=T^{\prime} \cup T^{\prime \prime}\right)$ will be forbidden in the SOC state if the corresponding subconfigurations on $T^{\prime}$ and $T^{\prime \prime}$ are both weak.

Let $T$ be a subtree of the lattice with root vertex $a$. Let the nearest neighbours of $a$ in $T$ be $a_{1}$ and $a_{2}$. Then on deleting the vertex $a, T$ breaks up into two subtrees $T_{1}$ and $T_{2}$ with roots $a_{1}$ and $a_{2}$ respectively. Let $N_{\mathrm{w}}(T, i)$ be the number of distinct weakly allowed subconfigurations on $T$ given that the height variable at the root $a$ has the value $i(1 \leq i \leq 3)$. Similarly we define $N_{s}(T, i)$ to be the number of distinct strongly allowed subconfigurations on $T$ with height variable $i$ at the root $a$. Also, let

$$
N_{\mathrm{w}}(T)=\sum_{i=1}^{3} N_{\mathrm{w}}(T, i)
$$

and

$$
N_{\mathrm{s}}(T)=\sum_{i=1}^{3} N_{\mathrm{s}}(T, i)
$$

For each subconfiguration that contributes to $N_{\mathrm{w}}(T, 1)$, there is a unique strongly allowed subconfiguration on $T_{1}$ and a unique strongly allowed subconfiguration on $T_{2}$ (obtained by deleting the root vertex $a$ ) and vice versa. This shows that

$$
N_{\mathrm{w}}(T, 1)=N_{\mathrm{s}}\left(T_{1}\right) N_{\mathrm{s}}\left(T_{2}\right)
$$


Arguing similarly, we get the following recursion relations:

$$
\begin{aligned}
& N_{\mathrm{w}}(T, 2)=N_{\mathrm{s}}\left(T_{1}\right) N_{\mathrm{w}}\left(T_{2}\right)+N_{\mathrm{w}}\left(T_{1}\right) N_{\mathrm{s}}\left(T_{2}\right) \\
& N_{\mathrm{w}}(T, 3)=N_{\mathrm{w}}\left(T_{1}\right) N_{\mathrm{w}}\left(T_{2}\right) \\
& N_{\mathrm{s}}(T, 1)=0 \\
& N_{\mathrm{s}}(T, 2)=N_{\mathrm{s}}\left(T_{1}\right) N_{\mathrm{s}}\left(T_{2}\right) \\
& N_{\mathrm{s}}(T, 3)=N_{\mathrm{s}}\left(T_{1}\right) N_{\mathrm{s}}\left(T_{2}\right)+N_{\mathrm{w}}\left(T_{1}\right) N_{\mathrm{s}}\left(T_{2}\right)+N_{\mathrm{s}}\left(T_{1}\right) N_{\mathrm{w}}\left(T_{2}\right)
\end{aligned}
$$

Adding (3.3), (3.4) and (3.5) and similarly (3.6), (3.7) and (3.8) we get

$$
\begin{aligned}
& N_{\mathrm{w}}(T)=\left[N_{\mathrm{s}}\left(T_{1}\right)+N_{\mathrm{w}}\left(T_{1}\right)\right]\left[N_{\mathrm{s}}\left(T_{2}\right)+N_{\mathrm{w}}\left(T_{2}\right)\right] \\
& N_{\mathrm{s}}(T)=2 N_{\mathrm{s}}\left(T_{1}\right) N_{\mathrm{s}}\left(T_{2}\right)+N_{\mathrm{w}}\left(T_{1}\right) N_{\mathrm{s}}\left(T_{2}\right)+N_{\mathrm{s}}\left(T_{1}\right) N_{\mathrm{w}}\left(T_{2}\right)
\end{aligned}
$$

Defining

$$
X(T)=\frac{N_{\mathrm{w}}(T)}{N_{\mathrm{s}}(T)}
$$

we find that it satisfies the following recursion relation:

$$
X(T)=\frac{\left[1+X\left(T_{1}\right)\right]\left[1+X\left(T_{2}\right)\right]}{2+X\left(T_{1}\right)+X\left(T_{2}\right)}
$$

This equation allows us to determine $X(T)$ recursively for any subtree $T$.
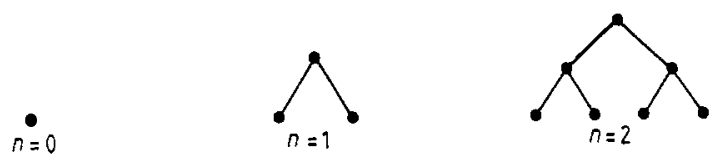

Figure 4. The recursive definition of $n$th generation binary trees for $n=0,1,2$.

Let $B_{n}$ be a binary tree of generation $n$. These are illustrated recursively in figure 4 . The graph $B_{0}$ consists of a single vertex. Clearly

$$
\begin{aligned}
& N_{\mathrm{w}}\left(B_{0}\right)=1 \\
& N_{\mathrm{s}}\left(B_{0}\right)=2
\end{aligned}
$$

and hence

$$
X\left(B_{0}\right)=\frac{1}{2}
$$

Equation (3.12) then simplifies to

$$
X\left(B_{n}\right)=\frac{1+X\left(B_{n-1}\right)}{2}
$$


with the initial condition given by (3.15). This has the simple solution

$$
X\left(B_{n}\right)=1-2^{-(n+1)} \text {. }
$$

As $n \rightarrow \infty, X\left(B_{n}\right)$ tends to 1 geometrically fast. It is easy to see that the argument can be extended to more general trees $T$ and in the limit when the distance of the root to the nearest surface site tends to infinity, we would get $X(T)=1$. Then from (3.3)-(3.8) we see that

$N_{\mathrm{w}}(T, 1): N_{\mathrm{w}}(T, 2): N_{\mathrm{w}}(T, 3): N_{\mathrm{s}}(T, 1): N_{\mathrm{s}}(T, 2): N_{\mathrm{s}}(T, 3)=1: 2: 1: 0: 1: 3$.

For finite trees, the root site is at a finite distance from the surface. In that case, there are (exponentially small with distance) corrections to this result. In the following, we shall ignore all such surface corrections.

\section{Single-site height distribution function}

We now calculate, in the SOC state, the probability distribution of heights at a randomly chosen site $O$ deep inside the lattice. This distribution has been obtained independently in [20] without, however, any explanation. Let $P(i)$ be the probability that the height at site $O$ is $i(1 \leq i \leq 3)$. Let the three subtrees connected to $O$ be $T_{1}, T_{2}$ and $T_{3}$ respectively (figure 5 ). We shall write $X\left(T_{j}\right)=x_{j}$ for simplicity of notation.

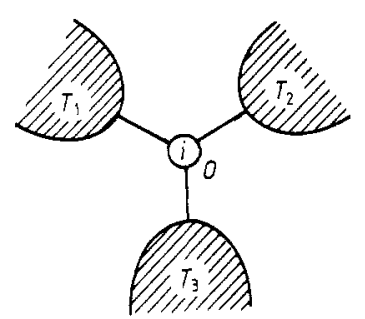

Figure 5. Figure showing the central site $O$ connected to three subtrees $T_{1}, T_{2}$ and $T_{3}$. Depending on the height $i(1 \leq i \leq 3)$ at $O$, only some of the allowed subconfigurations on $T_{i}$ give an allowed configuration on the full lattice.

Suppose $i=1$. Then, for a configuration to be allowed in the soc state, each of the subconfigurations on $T_{i}(i=1,2,3)$ should be strongly allowed. Thus, the number $N(1)$ of allowed configurations in SOC with $i=1$ at $O$ is given by

$$
N(1)=\prod_{i=1}^{3} N_{\mathbf{s}}\left(T_{i}\right)
$$

If $i=2$, at most one of the subtrees $T_{1}, T_{2}, T_{3}$, can be of the weakly allowed type. Therefore, the number of allowed configurations $N(2)$ with $i=2$ at $O$, in SOC, is

$$
N(2)=\left(1+\sum_{j=1}^{3} x_{j}\right) \prod_{i=1}^{3} N_{s}\left(T_{i}\right)
$$


A similar argument shows that

$$
N(3)=\left(1+\sum_{j=1}^{3} x_{j}+\sum_{j<k} x_{j} x_{k}\right) \prod_{i=1}^{3} N_{s}\left(T_{i}\right)
$$

Hence the total number of allowed configurations in the SOC state is given by

$$
N_{\text {total }}=\sum_{i=1}^{3} N(i)
$$

The probability $P(i)$ of having height $i$ at site $O$ is then given by, for $i=1,2,3$,

$$
P(i)=N(i) / N_{\text {total }} \text {. }
$$

When the site $O$ is sufficiently far from the surface of the lattice we have $x_{j}=1$ for all $j$. Then (4.4) reduces to

$$
N_{\text {total }}=12 \prod_{i=1}^{3} N_{\mathrm{s}}\left(T_{i}\right)
$$

Hence, from (4.5), we get

$$
P(1)=\frac{1}{12} \quad P(2)=\frac{4}{12} \quad P(3)=\frac{7}{12} .
$$

The average height at any site in the soc state is given by

$$
\langle i\rangle=\sum_{i=1}^{3} i P(i)=\frac{5}{2}
$$

\section{The pair distribution function in the soc state}

Consider two nearest-neighbour sites $A$ and $B$ deep inside the lattice (figure 6 ). Let $P(i, j)$ be the probability that the height variables at sites $A$ and $B$ are $i$ and $j$ respectively in the SOC state. Let the four subtrees that constitute the rest of the lattice be $T_{1}, T_{2}, T_{3}$ and $T_{4}$. Clearly, there are no configurations with $i=1$ and $j=1$ in the SOC state as this is a forbidden subconfiguration. Thus, we start with configurations for which $i=1$ and $j=2$. For these configurations to be allowed in the SOC state, all the subconfigurations on the four subtrees $T_{1}, T_{2}, T_{3}$ and $T_{4}$ have to be strongly allowed. The number of such configurations $N_{1,2}$ is clearly given by

$$
N_{1,2}=\prod_{i=1}^{4} N_{s}\left(T_{i}\right)
$$

In a similar way, we can determine the number of allowed configurations for any other value of $i$ and $j$. For example, in figure 7 are shown all distinct types of configurations 


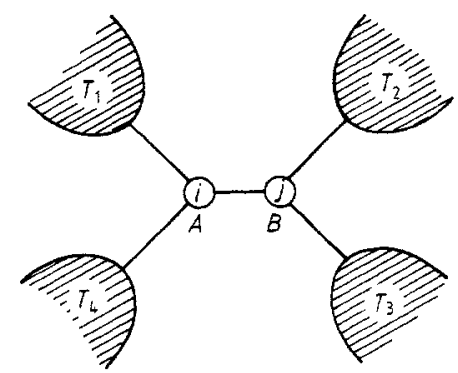

Figure 6. Figure showing adjacent sites $A$ and $B$ connected to four subtrees $T_{1}, T_{2}$, $T_{3}$ and $T_{4}$. The fact that $A$ and $B$ have heights $i$ and $j$ restrict the subconfigurations to be allowed on $T_{i}$.
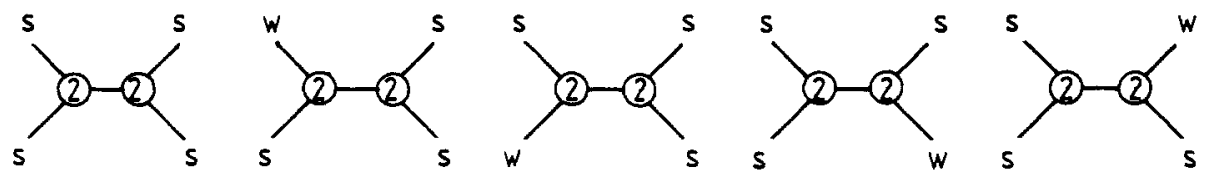

Figure 7. The five distinct types of subconfigurations allowed on the both have heights 2 .

that are allowed in SOC state with $i=2$ and $j=2$. Thus the number of allowed configurations of the full lattice with $i=2$ and $j=2$, is given by

$$
N_{2,2}=\left(1+\sum_{j=1}^{4} x_{j}\right) \prod_{i=1}^{4} N_{\mathrm{s}}\left(T_{i}\right)=5 \prod_{i=1}^{4} N_{\mathrm{s}}\left(T_{i}\right)
$$

The last step again uses the fact that all $x_{j}=1$ for sites deep inside the lattice.

For other values of $i$ and $j$, it is easy to write down similar expressions. The probabilities $P(i, j)$ are proportional to these numbers and the proportionality constant is determined by ensuring that the sum of all probabilities is unity. The product of the $N_{\mathrm{s}}\left(T_{i}\right)$ cancels with the same term in the normalisation constant. Thus, when both $A$ and $B$ are sufficiently far from the surface of the lattice, we get

$$
\begin{array}{ll}
P(1,1)=0 & P(1,2)=P(2,1)=\frac{1}{48} \\
P(2,2)=\frac{5}{48} & P(1,3)=P(3,1)=\frac{3}{48} \\
P(3,3)=\frac{15}{48} & P(2,3)=P(3,2)=\frac{10}{48} .
\end{array}
$$

This gives

$$
\langle i j\rangle=\sum_{i, j=1}^{3} i j P(i, j)=\frac{99}{16}
$$

which is a bit less than the value $\langle i\rangle\langle j\rangle=\frac{25}{4}$, indicating that the height variables in the SOC state are weakly anticorrelated.

In exactly the same way we can calculate the two-point correlation function of two sites $A_{1}$ and $A_{n+1}$ separated by distance $n$ (figure 8 ). The path from $A_{1}$ to $A_{n+1}$ goes through the points labelled $A_{1}, A_{2}, \ldots, A_{n}$. These sites $\left(A_{1}, \ldots, A_{n+1}\right)$ 
are connected to the rest of the lattice by $(n+3)$ subtrees $U_{1}, \ldots, U_{n+3}$. Then, for a given subconfiguration of heights at $\left(A_{1}, \ldots, A_{n+1}\right)$, the total number of allowed configurations in the SOC state of the full lattice can be calculated in terms of $N_{\alpha}\left(U_{k}\right)$, where $\alpha=w, s$ and $k=1,2, \ldots, n+3$. To determine the correlation function $P_{n}(i, j)$, i.e. the probability that the height at $A_{1}$ is $i$ and at $A_{n+1}$ is $j$, one has to, in addition, sum over different possibilities of heights at the intermediate sites $A_{k}$ with $k=2, \ldots, n$. A brute force calculation becomes rather tedious even for $n=2$. Luckily, it simplifies considerably in terms of a transfer matrix.
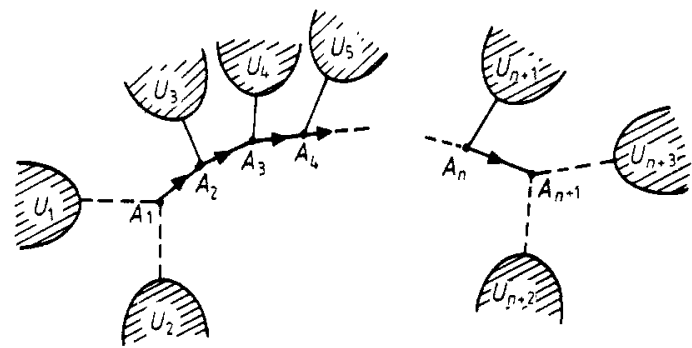

Figure 8. Two sites $A_{1}$ and $A_{n+1}$ of the lattice at a distance $n$. The path from $A_{1}$ to $A_{n+1}$ goes through the points $A_{2}, A_{3}, \ldots, A_{n}$. The rest of the lattice breaks up into subtrees $U_{1}, U_{2}, \ldots, U_{n+3}$. The arrows indicate the direction of transfer for the transfer matrix.

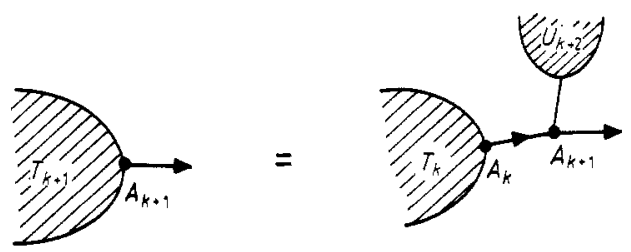

Figure 9. The recursive definition of subtrees $T_{k}$. The tree $T_{k+1}$ consists of the root $A_{k+1}$ and the subtrees $T_{k}$ and $U_{k+2}$ connected to $A_{k+1}$.

We define the subtree $T_{k}(k=1,2, \ldots, n)$ as the tree rooted at $A_{k}$ which gets disconnected from $A_{k+1}$ when the link connecting $A_{k}$ to $A_{k+1}$ is removed. We consider the number of allowed configurations of $T_{k}$ when the height variable at $A_{1}$ is held fixed at the value $i$. Then the tree $T_{k+1}$ consists of the root $A_{k+1}$ and the subtrees $T_{k}$ and $U_{k+2}$ (figure 9). Using (3.9) and (3.10), we get, for $k=1,2, \ldots, n-1$,

$$
\left(\begin{array}{c}
\bar{N}_{w}\left(T_{k+1}\right) \\
\bar{N}_{\mathrm{s}}\left(T_{k+1}\right)
\end{array}\right)=N_{\mathrm{s}}\left(U_{k+2}\right)\left(\begin{array}{cc}
1+x_{k+2} & 1+x_{k+2} \\
1 & 2+x_{k+2}
\end{array}\right)\left(\begin{array}{c}
\bar{N}_{\mathrm{w}}\left(T_{k}\right) \\
\bar{N}_{\mathrm{s}}\left(T_{k}\right)
\end{array}\right)
$$

where the bars above $N_{\mathrm{w}}\left(T_{k}\right)$ and $N_{\mathrm{s}}\left(T_{k}\right)$ denote the constraint that the height at $A_{1}$ is fixed at $i$. Using $x_{k}=1$ for all $k$ when the sites are far away from the surface of the lattice and

$$
\left(\begin{array}{c}
\bar{N}_{\mathrm{w}}\left(T_{1}\right) \\
\bar{N}_{\mathrm{s}}\left(T_{1}\right)
\end{array}\right)=\left(\begin{array}{c}
N_{\mathrm{w}}\left(T_{1}, i\right) \\
N_{\mathrm{s}}\left(T_{1}, i\right)
\end{array}\right)
$$

we get

$$
\left(\begin{array}{c}
\bar{N}_{w}\left(T_{n}\right) \\
\bar{N}_{\mathrm{s}}\left(T_{n}\right)
\end{array}\right)=\prod_{k=1}^{n-1} N_{\mathrm{s}}\left(U_{k+2}\right)\left(\begin{array}{ll}
2 & 2 \\
1 & 3
\end{array}\right)^{n}\left(\begin{array}{c}
N_{w}\left(T_{1}, i\right) \\
N_{\mathrm{s}}\left(T_{1}, i\right)
\end{array}\right)
$$


The total number of configurations, whose height at $A_{n+1}$ and at $A_{1}$ are fixed at $i$ and $j$ respectively, can be determined as in section 4 with $T_{1}, T_{2}$ and $T_{3}$ replaced by $T_{n}, U_{n+2}$ and $U_{n+3}$. For example, if $j=1$, the total number of allowed configurations with $j=1$ and height $i$ at $A_{1}$, is given by, from (4.1),

$$
N_{\text {total }}(i, j=1)=\bar{N}_{\mathbf{s}}\left(T_{n}\right) N_{\mathbf{s}}\left(U_{n+2}\right) N_{\mathbf{s}}\left(U_{n+3}\right)
$$

with $\vec{N}_{\mathrm{s}}\left(T_{n}\right)$ given by (5.7). We have to divide this number by the total number of allowed configurations of the full lattice in the SOC state, i.e. $N_{\text {total }}$ as given by (4.6) with $T_{1}, T_{2}$ and $T_{3}$ replaced by $T_{n}, U_{n+2}$ and $U_{n+3}$. Thus

$$
N_{\text {total }}=12 N_{\mathrm{s}}\left(T_{n}\right) N_{\mathrm{s}}\left(U_{n+2}\right) N_{\mathrm{s}}\left(U_{n+3}\right)
$$

where $N_{\mathrm{s}}\left(T_{n}\right)$ is obtained from $(5.7)$ by replacing $N_{\mathrm{w}}\left(T_{1}, i\right)$ and $N_{\mathrm{s}}\left(T_{1}, i\right)$ by $N_{\mathrm{w}}\left(T_{1}\right)$ and $N_{\mathbf{s}}\left(T_{1}\right)$ respectively. This gives

$$
N_{\text {total }}=3\left(4^{n+1}\right) \prod_{k=1}^{n+3} N_{s}\left(U_{k}\right)
$$

The eigenvalues of the $(2 \times 2)$ matrix in $(5.7)$ are 4 and 1 . Then, with a little algebra, we find that

$$
P_{n}(i, j)=\frac{N_{\text {total }}(i, j)}{N_{\text {total }}}=P(i) P(j)+f_{i, j} 4^{-n}
$$

where $P(i)(1 \leq i \leq 3)$ are the single-site distribution functions calculated in section 4 and the $f_{i, j}$ are numerical constants given by

$$
\begin{aligned}
& f_{1,1}=f_{1,2}=f_{2,1}=f_{2,2}=-\frac{1}{36} \\
& f_{1,3}=f_{2,3}=f_{3,2}=f_{3,1}=\frac{1}{18} \\
& f_{3,3}=-\frac{1}{9} .
\end{aligned}
$$

We see that correlations decay with distance as $4^{-n}$. Since the number of sites at distance $n$ grows only as $2^{n}$, we see that even the integral of these correlations is finite. This shows that correlation of height in the sOC state is effectively short-ranged on the Bethe lattice.

This is reminiscent of the conventional Bernoulli percolation problem, where the occupancy at different sites is uncorrelated. However, correlation functions depending on connectivity have a non-trivial dependence on distance. In our problem, the connectivity rule is given by the avalanche dynamics (whether a disturbance at site $i$ causes toppling at site $j$ ). The analogy to the percolation problem has been noted earlier $[1,20]$. However, Obukhov has argued [28] that the upper critical dimension for BTW-like models of SOC is 4 , and not 6 as in conventional uncorrelated percolation. On the Bethe lattice, we can show a precise correspondence between SOC and percolation problems. This we proceed to do in the next section. 


\section{The avalanche mass distribution function}

If a particle is added at a particular site $O$ of the lattice, the site will topple iff its height is 3 . As a result of this toppling at $O$, each of its three nearest neighbours will get one particle each. If any of these neighbours had height 3 already, it would topple in turn and the avalanche would continue. We shall define the mass of an avalanche as the number of distinct sites at which toppling occurs in the avalanche. It is easy to see that on the Bethe lattice, the cluster of toppled sites in an avalanche is the same as the connected set of all sites having height 3 including the site where a new particle has been added. Thus, the mass of the avalanche is equal to the number of sites in the corresponding connected cluster. For the Bethe lattice, it is thus easy to calculate the probability $g(n)$ that an avalanche has mass exactly equal to $n$ when a particle is added at $O$.

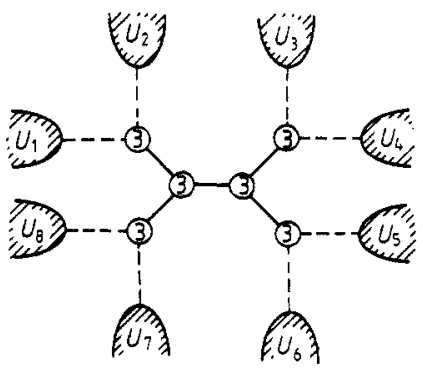

Figure 10. A connected cluster of sites with height 3 and size $n=6$. The rest of the lat tice breaks up into subtrees $U_{1}, U_{2}, \ldots, U_{8}$.

Consider a specific cluster $C$ (including $O$ ) of mass $n$, each site of the cluster having height 3 . The probability $U_{c}(n)$ of its occurrence in the SOC state can be computed as follows. The $n$ sites of the cluster are connected to the rest of the lattice by $(n+2)$ subtrees labelled $U_{1}, U_{2}, \ldots, U_{n+2}$ (figure 10). For a configuration of the full lattice containing $C$, to be allowed in the SOC state, there are two restrictions on the subconfigurations on the $U_{i}$ :

(i) the subconfigurations on the $U_{i}(i=1, \ldots, n+2)$ cannot be all weakly allowed;

(ii) the root of any of the $U_{i}$ cannot have height 3 .

The number of allowed configurations $N(C)$, containing $C$ and satisfying the above two restrictions is given by

$N(C)=\prod_{i=1}^{n+2}\left[N_{\mathrm{s}}\left(U_{i}, 2\right)+N_{\mathrm{w}}\left(U_{i}, 1\right)+N_{\mathrm{w}}\left(U_{i}, 2\right)\right]-\prod_{i=1}^{n+2}\left[N_{\mathrm{w}}\left(U_{i}, 1\right)+N_{\mathrm{w}}\left(U_{i}, 2\right)\right]$.

Using equation (3.18), we get, when the cluster is far away from the surface of the lattice,

$$
N(C)=\left[1-\left(\frac{3}{4}\right)^{n+2}\right] \prod_{i=1}^{n+2} N_{s}\left(U_{i}\right)
$$

To compute $U_{c}(n)$, we have to divide $N(C)$ by $N_{\text {total }}$, the total number of allowed configurations of the full lattice in the SOC state. Using arguments similar to that 
used in deriving (5.10), we get (with $n$ replaced by $(n+1)$ )

$$
N_{\text {total }}=3\left(4^{n}\right) \prod_{i=1}^{n+2} N_{s}\left(U_{i}\right)
$$

Hence

$$
U_{c}(n)=\frac{N(C)}{N_{\text {total }}}=\frac{1}{3}\left[1-\left(\frac{3}{4}\right)^{n+2}\right] 4^{-n} .
$$

Thus, $U_{c}(n)$ depends on the size $n$ of the cluster and not on its shape or perimeter. Hence we can drop the subscript $c$ in $U_{c}(n)$. Clearly then

$$
g(n)=U(n) a_{n}
$$

where $a_{n}$ is the number of distinct $n$-sized animals each including the site $O$. The computation of $a_{n}$ is well known for the Bethe lattice [29], but for the sake of completeness we repeat it here. We define the generating function $G(x)$ of $a_{n}$ as follows

$$
G(x)=\sum_{n=1}^{\infty} a_{n} x^{n}
$$

The site $O$ is connected to the rest of the lattice by subtrees $T_{1}, T_{2}$ and $T_{3}$ (figure 5 ).

Let $B(x)$ denote the number of lattice animals on any one of the subtrees $T_{i}$

$$
B(x)=\sum_{n=1}^{\infty} b_{n} x^{n}
$$

where $b_{n}$ is the number of $n$-sized clusters on the subtree $T_{i}$.

Then, $B(x)$ and $G(x)$ satisfy the simple equations

$$
B(x)=x[1+B(x)]^{2}
$$

and

$$
G(x)=x[1+B(x)]^{3}
$$

solving (6.8) for $B(x)$, we get

$$
B(x)=\frac{1-2 x-\sqrt{1-4 x}}{2 x}
$$

and

$$
G(x)=\frac{1}{8 x^{2}}(1-\sqrt{1-4 x})^{3} .
$$

Expanding equation (6.11) in powers of $x$, we get

$$
a_{n}=\frac{3}{2 n+1}^{2 n+1} C_{n-1}
$$


Substituting (6.4) and (6.12) in (6.5), we get

$$
g(n)=\frac{1}{2 n+1}^{2 n+1} C_{n-1}\left[1-\left(\frac{3}{4}\right)^{n+2}\right] 4^{-n}
$$

For large $n$, this gives

$$
g(n) \sim n^{-3 / 2}
$$

Thus the avalanche mass has an asymptotic power-law distribution for large mass which is a typical signature of the SOC. The exponent $\frac{3}{2}$ is the same as that which characterises the cluster-size distribution in an ordinary (uncorrelated) percolation problem in high dimensions. Thus, at least on the Bethe lattice, small anticorrelations in height variables have no significant effect. They shift the critical value of the concentration of 3 's from its uncorrelated percolation value $\frac{1}{2}$ to a somewhat higher value $\frac{7}{12}$, but do not result in a change of the critical exponents.

From (6.14), the probability that the avalanche mass exceeds $n$ varies as $n^{-1 / 2}$ for large $n$. Since in our problem, all avalanches of $n$ sites (irrespective of their shapes) have equal probability of occurrence, the average space extent of an avalanche of size $n$ is exactly same as that of a random animal of $n$ sites. On the Bethe lattice, it is well known $[30,31]$ that the typical extent of an animal of size $n$ (as measured by the distance of the farthest point of the animal measured along the bonds of the lattice) varies as $n^{1 / 2}$ for large $n$. This implies that the probability that an avalanche created by adding a particle at a random site will travel a distance $r \geq R$ along bonds is given by, for large $R$,

$$
\operatorname{Prob}(r>R) \sim \frac{1}{R} .
$$

If we define the Euclidean distance between two sites separated by $R$ steps along the bonds of the lattice as $R_{\mathrm{E}}=\sqrt{R}$, then the probability that an avalanche extends at least up to Euclidean distance $R_{\mathrm{E}}$ varies as $R_{\mathrm{E}}^{-2}$ for large $R_{\mathrm{E}}$.

We note that the largest distance along bonds is related to the duration of an avalanche. To be specific, it is easy to show that the duration $T$ of an avalanche satisfies the inequality $R<T \leq 2 R$, where $R$ is the maximal distance along bonds travelled by the avalanche. This implies, from $(6.15)$ that, for large $T$,

$$
\text { Prob(duration }>T) \sim \frac{1}{T} \text {. }
$$

\section{Distribution of toppling numbers}

If a particle is added at a particular site $O$ on the lattice, the site might topple more than once depending upon the height configuration of its neighbours. Note that the site $O$ would topple at least $n$ times iff all the sites up to distance $(n-1)$ all have height 3 at the time the particle is added. Let $N_{n}$ be the number of allowed configurations of the full lattice such that adding a particle at $O$ causes $n$ or more topplings at $O$. In each such cluster, all the heights up to a distance $(n-1)$ are all 3 , and these are 
connected to the rest of the lattice by $3\left(2^{n-1}\right)$ subtrees $T_{1}, T_{2}, \ldots, T_{3\left(2^{n-1}\right)}$. The only restriction is that these subtrees should not all have weakly allowed configurations. This gives

$$
N_{n}=\prod_{i=1}^{3\left(2^{n-1}\right)}\left[N_{\mathrm{w}}\left(T_{i}\right)+N_{\mathbf{s}}\left(T_{i}\right)\right]-\prod_{i=1}^{3\left(2^{n-1}\right)} N_{\mathrm{w}}\left(T_{i}\right)
$$

and using $x\left(T_{i}\right)=1$ we get

$$
N_{n}=\left(2^{3\left(2^{n-1}\right)}-1\right) \prod_{i=1}^{3\left(2^{n_{1}}\right)} N_{\mathrm{s}}\left(T_{i}\right)
$$

The total number of allowed configurations in the SOC state can also be expressed in terms of the $N_{\mathbf{s}}\left(T_{i}\right)$ by the same argument as used in deriving (5.10)

$$
N_{\text {total }}=3\left(4^{3\left(2^{n-1}-2\right)}\right) \prod_{i=1}^{3\left(2^{n-1}\right)} N_{\mathrm{s}}\left(T_{i}\right) .
$$

Hence $f(n)$, the probability that the origin $O$ topples at least $n$ times, is given by

$$
f(n)=N_{n-1} / N_{\text {total }}=\left(\frac{16}{3}\right) 2^{-3\left(2^{n-1}\right)}\left[1-2^{-3\left(2^{n-1}\right)}\right] .
$$

We see that $f(1)=\frac{7}{12}, f(2)=\frac{21}{256}$, etc. For large $n, f(n)$ decays to 0 extremely rapidly with $n$ (as an exponential of an exponential). This indicates that multiple topplings at a particular site on the Bethe lattice is a rare event in the soc state.

Since a site might topple more than once before the system comes to a stable configuration, the number of distinct toppled sites in an avalanche is not the same as the number of topplings in the avalanche. Below we calculate the toppling number distribution function $F(s)$, i.e. the probability that there will be in total exactly $s$ topplings in the system.

We consider an avalanche cluster of $n$ distinct toppled sites, for which the origin topples exactly $r$ times. Then it follows that its three nearest neighbours will each topple exactly $(r-1)$ times, the six next-nearest neighbours will each topple exactly $(r-2)$ times, etc. All the sites at distance $(r-2)$ will topple exactly twice, and all the sites with distance greater than $(r-2)$ will topple exactly once. Adding, this gives us the total number of topplings $s$ in the avalanche as

$$
s=n+3\left(2^{r-1}\right)-2 r-1 .
$$

Let $A_{n, r}$ be the number of distinct clusters of $n$ sites such that the origin topples $\geq r$ times. We define the generating function

$$
A_{r}(x)=\sum_{n=1}^{\infty} A_{n, r} x^{n} .
$$

Then it is easy to see that for $r \geq 1$

$$
A_{r}(x)=\frac{1}{x^{2}}[x(1+B(x))]^{3\left(2^{r-1}\right)}
$$


with $B(x)$ given by $(6.10)$.

Now the number of clusters having $n$ distinct sites where the origin topples exactly $r$ times is $\left(A_{n, r}-A_{n, r+1}\right)$. From (6.4), the probability of occurrence of any one of these in the soC state is $\left(1-\left(\frac{3}{4}\right)^{n+2}\right) 4^{-n} / 3$. Let $P_{s}$ be the probability that there are exactly $s$ topplings in the avalanche. Then using (7.5) we get

$$
\begin{aligned}
P(x)=\sum_{s=1}^{\infty} P_{s} x^{s}= & \frac{1}{3} \sum_{n, r}\left(A_{n, r}-A_{n, r+1}\right)\left[1-(3 / 4)^{n+2}\right] 4^{-n} x^{n+3\left(2^{r-1}\right)-2 r-1} \\
= & \frac{1}{3} \sum_{r=1}^{\infty}\left[A_{r}(x / 4)-A_{r+1}(x / 4)-\frac{9}{16} A_{r}(3 x / 16)\right. \\
& \left.+\frac{9}{16} A_{r+1}(3 x / 16)\right] x^{3\left(2^{r-1}\right)-2 r-1} .
\end{aligned}
$$

It seems difficult to get a closed-form formula for $P_{s}$. However, the series is rapidly convergent in $r$ for fixed $x<1$ and each of the functions $A_{r}(x)$ has a singularity of the type $(1-x)^{1 / 2}$. It thus follows that

$$
P_{s} \sim s^{-3 / 2}
$$

for large $s$. This result might have been expected a priori, as multiple topplings are rare and so $s$ is roughly same as the number of distinct sites toppled.

\section{Generalisation to $(q+1)$ coordinated Bethe lattices}

So far we have been discussing the height model of SOC on a $(q+1)$ coordinated Bethe lattice with $q=2$. The model can be generalised to a $(q+1)$ coordinated Bethe lattice with $q \geq 2$. The critical height at any site, in the general case, is $(q+1)$ and if the height exceeds $(q+1)$ a toppling occurs at that site. The treatment is a straightforward generalisation of the $q=2$ case, but the algebra becomes more tedious. We mention a few of the results.

Equation (3.17) for the ratio of the number of weak and strong configurations, generalises to

$$
X\left(B_{n}\right)=\frac{1}{q-1}\left(1-q^{-(n+1)}\right) .
$$

As $n \rightarrow \infty, X\left(B_{n}\right)$ tends to $1 /(q-1)$.

The single-site height distribution function $P(i)(1 \leq i \leq q+1)$ is given by

$$
P(i)=\frac{1}{\left(q^{2}-1\right) q^{q}} \sum_{m=0}^{i-1}{ }^{q+1} C_{m}(q-1)^{q-m+1} .
$$

For $q=2,3,4$ these results have been obtained independently by Grassberger and Manna [20].

The avalanche mass distribution function $g(n)$ can be calculated similarly. The expression for $U(n)$, as defined in (6.5), is given by

$$
U(n)=\frac{(q-1)^{n(q-1)+2}-\left(1-q^{-q}\right)^{n(q-1)+2}}{\left(q^{2}-1\right) q^{n q}} .
$$


The number of distinct $n$-sized animals $a_{n}$, each including the origin, was originally calculated by Fisher and Essam [29] for a $(q+1)$ coordinated Bethe lattice and is given by

$$
a_{n}=\frac{q+1}{n q+1}^{n q+1} C_{n_{1}} .
$$

Combining (8.3) and (8.4) in (6.5), we get for large $n$

$$
g(n)=U(n) a_{n} \sim n^{-3 / 2} .
$$

Thus the exponent of the power-law distribution of avalanche mass is still $\frac{3}{2}$ and is independent of $q$.

\section{Discussion}

In this paper we have determined the critical exponents of the Abelian sandpile model of SOC on the Bethe lattice, and have shown that they agree with the mean-field critical exponents of percolation. These results would also be expected to hold for the more conventional $d$-dimensional spaces $Z^{d}$, when the dimension $d$ exceeds the upper critical dimension $d_{\mathrm{u}}$ for this problem. The value of $d_{\mathrm{u}}$ in this case is not quite settled. As mentioned earlier, it has been argued [28] that $d_{u}=4$, as this problem resembles a branching self-avoiding walk problem. The relationship to the percolation problem would suggest that $d_{u}=6$. However, this problem actually corresponds to a correlated percolation problem with very specific correlations and the value of $d_{u}$ could be lower.

We note that the fractal dimension of avalanche clusters is 4 on the Bethe lattice [32]. Assuming that this is true for all hypercubical lattices with dimension $d \geq d_{\mathrm{u}}$ as well, we obtain

$$
d_{\mathrm{u}} \geq 4
$$

as the fractal dimension of avalanche clusters must be lower than that of the embedding space. An interesting possibility is that for $d \geq 4$, the avalanche clusters have fractal dimension 4 , but for $d \leq 4$, they are compact. We note that the exponent value $\tau=\frac{3}{2}$ on the Bethe lattice agrees with Zhang's values for $d=4$ [16], derived using the assumption that the clusters are compact. We note, however, that for $d=2$, Zhang's argument gives $\tau=1$, a value which disagrees significantly with the value $\tau=1.22$ obtained in recent numerical simulations [20].

It seems rather unlikely that a simple general theory of SOC will be able to describe all the different kinds of models discussed in the introduction. One can only hope that study of some of these models will help us understand their criticality more clearly. With hindsight, the power-law describing, say, the distribution of avalanche masses in the sandpile model might appear as trivial as that of the power-law tails in the auto-correlation function of fluids. Until then, further study of some simple models seems necessary. In particular, the relationship between intermittency, chaos and SOC is quite intriguing and deserves further study. 


\section{Acknowledgments}

We thank Dr M Barma for a critical reading of the manuscript.

\section{References}

[1] Bak P, Tang C and Wisenfeld K 1987 Phys, Rev. Lett. 59381

- 1988 Phys. Rev. A 38364

[2] Tang C and Bak P 1988 J. Stat. Phys. 51797

Alstrom P 1988 Phys. Rev. A 384905

[3] Takayasu H, Nishikawa I and Tasaki H 1988 Phys. Rev. A 373110

[4] Parisi G 1988 Statistical Field Theory (Reading, MA: Addison Wesley) p 192

[5] Kadanoff L P 1990 Physica 163A 1

[6] Obhukov S P Preprint BNL

[7] Landau L D and Lifshitz E M 1989 Fluid Mechanics (Oxford: Pergamon) p 95

[8] Montroll E W and West B J 1973 Synergetics ed H Haken (Leipzig: Teubner) p 143

[9] Bak P and Chen K 1989 Physica 38D 5

[10] Hermann H J 1986 Phys. Rep. 136153

[11] Kardar M, Parisi G and Zhang Y C 1986 Phys. Rev. Lett. 56889

[12] Wilkinson D and Willemsen J F 1983 J. Phys. A: Math. Gen. 163365

[13] Binder K 1979 Monte Carlo Methods in Statistical Physics (Berlin: Springer)

[14] Carlson J M and Langer J S 1989 Phys. Rev. Lett. 622632

1989 Phys. Rev. A 406470

[15] Takayasu H 1989 Phys. Rev. Lett. 632563

[16] Zhang Y C 1989 Phys. Rev. Lett. 63470

[17] Hwa T and Kardar M 1989 Phys. Rev. Lett. 621813

1989 Physica 38D 198

[18] Kadanoff L P, Nagel S R, Wu L and Zhou S M 1989 Phys. Rev. A 396524

[19] Manna S S 1990 J. Stat. Phys. 59509

[20] Grassberger $P$ and Manna S S 1990 Preprint Wuppertal

[21] Carlson J M, Chayes J T, Grannan E R and Swindle G H 1990 Preprint ITP

[22] Wiesenfeld K, Theiler J and McNamara B 1990 Preprint Georgia Tech

[23] Creutz M 1990 Preprint BNL

[24] Dhar D 1990 Phys. Rev. Lett. 641613

[25] Dhar D and Ramaswamy R 1989 Phys. Rev. Lett. 631659

[26] McNamara B and Wiesenfeld K 1990 Phys. Rev. A 411867

[27] Baxter R J 1982 Exactly Solved Models in Statistical Mechanics (New York: Academic) ch 5

[28] Obukhov S P 1988 Random Fluctuations and Pattern Growth ed H E Stanley and N Ostrowsky (Dordrecht: Kluwer) p 336

Honkonen J 1990 Phys. Lett. 145A 87

[29] Fisher M E and Essam J W 1961 J. Math. Phys. 2609

[30] Lubensky T C and Isaacson J 1979 Phys. Rev. A 202130

[31] Daoud M and Joanny J F 1981 J. Physique 421359

[32] Stauffer D 1985 Introduction to Percolation Theory (London: Taylor and Francis) p 65 